sizes from national data are often used by researchers while actual portions are often much larger, and therefore include more calories. Finally, data on the food sources of nutrients are not publicly available. Current data describes food item sales yet nutrient quantities are not available for each item, preventing determination of company and food item sources of nutrient intake. To successfully improve the food environment and promote health through diet, measurement of dietary intake quality and supply must be improved.

\section{P1-127 SPATIAL AND TEMPORAL DYNAMICS OF DENGUE FEVER CASES IN ARARAQUARA CITY, BRAZIL}

doi:10.1136/jech.2011.142976d.20

M A De Oliveira, ${ }^{*}$ H Ribeiro, M Inenami, R G da Silva. Public Health School, University of São Paulo, São Paulo, Brazil

Background Dengue fever represents a serious public health issue in Brazil. Araraquara is a city, in state of São Paulo that has been having serious outbreaks of dengue fever in the last decade, in spite of its relatively good social and economical conditions. This study mapped and analysed spatial and time distribution of the cases of dengue fever in Araraquara.

Methods Registered and confirmed cases of years 2007 and 2008 from the National System of Diseases Notification (SINAN) were geo-referenced using a street based map and a Geographic Information System (GIS). To analyse spatial temporal distribution pattern of dengue fever cases, density Kernel were used on the events according to epidemiological week.

Results In 2007, 335 cases were confirmed, and in 2008, 1233 new cases. Spatial analysis presented occurrence of dengue fever cases almost all around the urban area of the municipality of Araraquara. Important spatial variations on the distribution pattern along the epidemiological weeks were identified. In the two analysed years, most areas that presented high concentration of cases in a specific week tended to have fewer cases on the following week. However, in some areas of the city were identified clusters of dengue fever that remained in the same place.

Conclusion The results showed that, at a local intra urban level, the analysis of cases per epidemiological week can be an way to identify spatial and time important differences on the incidence of the disease and possible factors associated.

\section{P1-128 A SYSTEMATIC REVIEW OF SEXUAL RISK FACTORS FOR HIV IN INDIA}

doi:10.1136/jech.2011.142976d.21

${ }^{1} \mathrm{~N}$ Dhingra, ${ }^{1} \mathrm{P}$ Arora, ${ }^{*}{ }^{1} \mathrm{P}$ Jha. ${ }^{1}$ National AIDS Control Organization, Ministry of Health and Family Welfare, Government of India, New Delhi, India; ${ }^{2}$ Centre fro Global Health Research, Li Ka Shing Knowledge Institute, St Michael's Hospital, Dalla Lana School of Public Health, University of Toronto, Toronto, Canada

Introduction India's large HIV epidemic and the potential for epidemic HIV spread in some areas demands a full understanding of HIV transmission. Factors associated with HIV infection in India include: commercial sex work, sexually transmitted infections (STI), numbers of sex partners and male circumcision. We wished to quantify effects of the above risk factors, roughly estimating their size, for each gender and in high- and low-HIV risk populations.

Methods Meta-analysis of risk factors for HIV from 34 studies identified through literature search of multiple on-line databases. Risk factors were: male circumcision/religion, biologically confirmed infection with Herpes Simplex Virus 2, syphilis or gonorrhoea, selfreported genital ulcer, multiple sexual partners and paid sex work. Random-effects meta-analysis of effects was conducted.

Results Each of the seven risk factors were significantly associated with HIV status in both men and women. The strongest effect size among men was HSV-2 infection (OR: 4.68; 95\% CI 2.40 to 5.43) while the strongest effect among women was ever being paid for sex (OR: 5.06 ; 95\% CI 3.69 to 6.95). The effect of multiple sexual partners was similar among men $(2.80$; $95 \%$ CI 2.09 to 3.77 ,) and women (3.00; 95\% CI 1.88 to 4.79) and was similar when further stratified by HIV-risk group. Syphilis and multiple sexual partners as risk factors for HIV were significantly associated with lower HIVrisk study populations.

Conclusions The risk factors examined in our analysis should remain targets of HIV prevention programs. Our results confirm that sexual risk factors for HIV infection remain an important part of the HIV epidemic in India.

\section{P1-129 THE IMPORTANCE OF REPRODUCTIVE HEALTH AND HIV/ AIDS EDUCATION PROGRAM FOR YOUNG PEOPLE IN PAPUA AND WEST PAPUA PROVINCES, INDONESIA}

doi:10.1136/jech.2011.142976d.22

1,2W Diarsvitri, * II Dwisetyani. 'Department of Community Health, Faculty of Medicine, Hang Tuah University, Surabaya, East Java, Indonesia; ${ }^{2}$ Australian Demographic and Social Research Institute, The Australian National University, Canberra, ACT, Australia

Introduction Papua and West Papua Provinces have the highest HIV/ AIDS prevalence in Indonesia. In 2009, 94.4\% HIV/AIDS cases in Papua Province were transmitted through heterosexual intercourse and the cases among 15-19 years age group were in the fourth rank. A study in 2007 revealed 46.9\% Junior High School students had misconception on HIV/AIDS transmission and prevention. These features motivated the author to carry out the 2009 Reducing the Risk of HIV/AIDS: Intervention Trial for Young Papuans Study. The aim of this study was to evaluate of the reproductive health and HIV/AIDS education program in changing young people's knowledge, attitudes and behaviour intention associated with HIV/AIDS and sexuality.

Materials and Methods Sixteen senior high schools were randomly selected to either receive the program or to act as a control group. Students of Year 11 from the selected schools $(n=1082)$ took a preintervention test and 2 months later, a post-intervention test. Changes in knowledge, attitude and behavioural intentions between the two groups were compared using a mixed model.

Results Findings showed that the intervention was associated with 0.11 points higher knowledge (95\% CI 0.08 to 0.12 ), 0.13 points higher attitude (95\% CI 0.09 to 0.22$)$ and 0.18 points higher behavioural intentions ( $95 \%$ CI 0.11 to 0.33 ) after adjusting for age, gender, previous sexual experience, ethnicity and pre test mean score. Conclusions Our reproductive health and HIV/AIDS education program was effective in changing knowledge, attitude and behavioural intentions of students. It is important to promote its inclusion in the school curricula.

\section{P1-130 CREUTZFELDT-JAKOB DISEASE: REPORT OF CASES}

doi:10.1136/jech.2011.142976d.23

\section{J Dias.* Secretary of State of Health of Bahia, Salvador, Bahia, Brazil}

Introduction Creutzfeldt-Jakob disease (CJD) is an infectious disease attributed to a proteinaceous infectious particle (prion), which attacks the central nervous system. The form of acquisition is still unknown and may occur by four known mechanisms of transmission: sporadic, hereditary, iatrogenic and variant (vCJD), which occurs by eating contaminated beef carrier of mad cow disease.

Methods Reporting of cases of CJD has occurred in Bahia between August 2007 and December 2010.

Results There have been 14 suspected cases of sporadic CJD, eight confirmed, two probable (WHO criteria), one discarded and three 
under investigation. Of these, six were male and white. The median age was 68 years old, ranging from 40 to 77 . Four had complete higher education, four high school and two elemetary education. Ten died. None of the suspected cases underwent iatrogenic exposure and had no family history of disease. All had cerebellar ataxia, $80 \%$ had progressive dementia and extra-pyramidal signs and $60 \%$ had psychiatric disorders, pyramidal signs, myoclonus and sleep disturbances. Of the specific tests performed five had typical result by electroencephalogram; six with typical diffusion by MRI/CT; five presented Protein 14-3-3; none of them presented mutation of prion; four patients had spongiform encephalopathy brain by biopsy; four positive by immunohistochemistry for PRPNPc. Only three patients underwent necropsy had histological positive result.

Conclusion Since this is a new and yet unknown disease in our country, needs to improve the clinical diagnosis as a way to better understand the natural history of it and thereby promote effective measure control.

\section{P1-131 THE IMPACT OF ATTRITION ON THE REPRESENTATIVENESS OF COHORT STUDIES OF OLDER PEOPLE}

doi:10.1136/jech.2011.142976d.24

A Dobson, ${ }^{*}$ S Brilleman, N Pachana. University of Queensland, Brisbane, Australia

Introduction There are well-established risk factors, such as lower education, for attrition of study participants. Consequently, the representativeness of the cohort in a longitudinal study may deteriorate over time. Death is a common form of attrition in cohort studies of older people. The aim of this paper is to examine the effects of death and other forms of attrition on risk factor prevalence in the study cohort and the target population over time.

Methods Differential associations between a risk factor and death and non-death attrition are considered under various hypothetical conditions and data from the Australian Longitudinal Study on Women's Health (ALSWH) and the Australian Censuses and National Health Surveys are used to illustrate the evolution of bias over 12 years.

Results Between 1996 and 2008, 28.4\% of ALSWH participants born in 1921-1926 died, 16.5\% withdrew and 10.4\% were lost to followup. Hypothetical scenarios illustrate how death and other attrition can affect changes in bias over time. For this cohort there were differential associations with various risk factors, for example, being born in a non-English speaking country was associated with nondeath attrition but not death whereas being underweight was associated with death but not other forms of attrition.

Conclusions Deaths occur in both the target population and study cohort, while other forms of attrition occur only in the study cohort. Therefore non-death attrition may cause greater bias than death in longitudinal studies. However although more than a quarter of the oldest participants in the ALSWH died, differences from the national population changed only slightly.

\section{P1-132 DESCRIPTION OF A NATIONWIDE AND MULTIDISCIPLINARY PROJECT FOR THE EVALUATION OF INTERVAL BREAST CANCER RATES, DETERMINANTS AND CHARACTERISTICS, IN SPAIN. INCA STUDY}

doi:10.1136/jech.2011.142976d.25

\footnotetext{
${ }^{1} \mathrm{M}$ Sala, ${ }^{1} \mathrm{~L}$ Domingo,* ${ }^{1} \mathrm{~J}$ Blanch, ${ }^{2} \mathrm{M}$ Baré, ${ }^{3} \mathrm{~J}$ Ferrer, ${ }^{4} \mathrm{~A}$ B Fernández, ${ }^{5} \mathrm{D}$ Salas, ${ }^{5} \mathrm{~J}$ lbáñez, ${ }^{6} \mathrm{G}$ Sarriugarte, ${ }^{7} \mathrm{~J} \mathrm{M}$ Reyes, ${ }^{8} \mathrm{M}$ Rué, ${ }^{1} \mathrm{X}$ Castells. ${ }^{1}$ Department of Epidemiology and Evaluation, IMIM-Parc de Salut Mar. CIBERESP, Barcelona, Spain; ${ }^{2}$ Epidemiology and Assessment Unit UDIAT-Diagnostic Center. Corporació Parc Tauli, Sabadell, Spain; ${ }^{3}$ Radiology Service, Hospital de Santa Caterina, Girona, Spain; ${ }^{4}$ Galician breast cancer screening programme. Public health \& Planning Directorate, Santiago de Compostela, Spain; ${ }^{5}$ General Directorate Public Health \& Centre for Public
}

Health Research, Valencia, Spain; ${ }^{6}$ Osakidetza Breast Cancer Screening Programme, Basque Country Health Service, Bilbao, Spain; ${ }^{7}$ General Directorate of Health Care Programmes, Canary Islands Health Service, Canary Islands, Spain; ${ }^{8}$ Basic Medical Sciences Department, Biomedical Research Institute of Lleida (IRBLLEIDA), Lleida, Spain

Introduction Interval cancer (IC) rate, risk factors and biological characteristics have been scarcely evaluated. In January 2010 started a multicentric project with the aim to estimate rates and determinants of IC and to compare their characteristics with those detected in the routine screening mammography.

Methods Information from women aged $45 / 50$ to 69, participating in seven population-based screening programs in Spain from January 2000 to December 2006, was collected. The cohort was followed-up to June 2009 for cancer (IC and screening) identification. Three protocols were defined: (1) To describe the joint database format and architecture, and for the achievement of variables, including women and tumour-related data (prognostic factors and biomarkers expression); (2) To unify IC diagnosis criteria and identification process; (3) A radiological protocol for mammogram's review and IC classification, based on European Guidelines.

Results A database with information of 1350058 mammographies from 759604 screened women has been built. $94.2 \%$ were analogical and $5.8 \%$ digital mammographies. A total of 4656 screen-detected cancers and 1479 IC have been identified. For IC identification, which is not finished, databases of screening programs have been linked with poblational and hospital-based cancer registries and data from active case search.

Conclusions The database will allow us to evaluate women's and screening determinants for IC (including true interval and false negatives cancers), and to compare tumour characteristics between IC and screen-detected cancers. This information will be useful to improve the efficiency of screening programs and the breast cancer classification, and could offer new insights on cancer prevention for specific subsets of women.

\section{P1-133 TOBACCO CONSUMPTION AMONG ADOLESCENTS IN RURAL INDIA: WHERE AND HOW TOBACCO CONTROL SHOULD FOCUS ITS ATTENTION?}

doi:10.1136/jech.2011.142976d.26

${ }^{1}$ A Dongre, ${ }^{* 2} \mathrm{P}$ Deshmukh, ${ }^{2} \mathrm{~N}$ Murali, ${ }^{2} \mathrm{~B}$ Garg. ${ }^{1}$ Department of Community Medicine, Sri Manakula Vinayagar Medical College and Hospital, Pondicherry, India; ${ }^{2}$ Dr Sushila Nayar School of Public Health, Mahatma Gandhi Institute of Medical sciences, Sewagram, India

Objectives The objectives of the present study were to study the pattern of tobacco use among rural adolescents (15-19 years) and to find out reasons for use and non use of tobacco products in rural central India

Material and Methods In the present community based research, triangulation of qualitative (free list, focus group discussions) and quantitative methods (survey) was undertaken. The findings of qualitative assessment and the Global Youth Tobacco Survey (GYTS) questionnaire were used for development of locally relevant questionnaire. Assuming the rough estimate of rural adolescents proportion, for knowledge and practices of study variables as 0.5 , $95 \% \mathrm{CI}$ and $5 \%$ precision, the minimum sample size required was 384 which was inflated by $10 \%$ to cover non-response. A team of trained medical personnel interviewed 385 adolescents (92\% response rate) by paying home visits. After survey, six focus group discussions were undertaken with adolescent boys to bridge gaps in information and develop a conceptual framework of pro-tobacco influences.

Results About $68.3 \%$ boys and $12.4 \%$ girls had consumed any tobacco products in last 30 days. Among boys, $51.2 \%$ consumed it 